\title{
Salar de Surire un ecosistema altoandino en peligro, frente a escenario del cambio climático
}

\author{
I. Garcés* \\ Departamento de Ingeniería Química, Universidad de Antofagasta \\ Av. Universidad de Antofagasta 02800, Antofagasta, Chile \\ E-mail: igarces@uantof.cl
}

(recibido/received: 14-Enero-2011; aceptado/accepted: 10-Junio-2011)

\begin{abstract}
RESUMEN
Este trabajo realiza un análisis al salar de Surire desde el punto de vista de la biodiversidad y sus implicancias producto del cambio climático. Como resultado se predice que los próximos años serán secos y la temperatura podría ir en aumento, lo cual afectará notablemente a la flora y fauna del altiplano. El salar de Surire es un cuerpo salino ubicado en el altiplano andino chileno, en un entorno geológico de características volcánicas. La climatología actual de aridez no permite una acumulación de agua superficial de gran extensión, y por lo tanto es posible diferenciar tres tipos principales de aguas: los aportes de drenaje, las acumulaciones de agua en la aureola pantanosa externa o "bofedales", y las aguas del interior del cuerpo salino (surgencias termales y salmueras). Estas condiciones hidrológicas afectan factores abióticos, como anaerobiosis de suelos, disposición de nutrientes y salinidad, factores determinantes del desarrollo de la flora y fauna.
\end{abstract}

Palabras claves: Salar de Surire; Biodiversidad andina; Cambio climático; Ecosistema andino

\begin{abstract}
In this work, the Surire salt marsh was analysed from the viewpoint of biodiversity and its implications caused by the climate change. As result, it is predicted that the following years will be dry and temperatures could rise, which will considerably affect the flora and fauna of the plateau. The Surire salt marsh is a salt body located in the Andean highlands of Chile, in a geological environment of volcanic features. The actual dryness does not allow an accumulation of surface water with large volume; thus, it is possible to identify three main types of water: drainage contributions, accumulation of external water or wetlands, and the thermal springs and brines. These hydrological conditions affect abiotic factors such as soil anaerobiosis, availability of nutrients and salinity, factors determining the development of flora and fauna.
\end{abstract}

Keywords: Surire salt marsh; Andean biodiversity; Climate change; Andean ecosystem

*Autor para la correspondencia 


\section{INTRODUCCIÓN}

Honty (2007) señala que la Convención Marco de las Naciones Unidas sobre Cambio Climático (CMNUCC) define el cambio climático como un cambio del clima atribuido directa $\mathrm{o}$ indirectamente a la actividad humana que altera la composición de la atmósfera mundial. Entonces el cambio climático es cualquier cambio significativo en las medidas del clima (tal como la temperatura o precipitación) que dura por un período extenso de tiempo. En este contexto, el presente trabajo que se circunscribe a un sistema salino altoandino, en el noreste de Chile, da cuenta del cambio en las precipitaciones altiplánicas que de acuerdo a los registros de los 40 últimos años, presenta una tendencia a la baja, observándose además el deshielo de glaciares en las altas cumbres, y el aumento en las temperaturas, que como consecuencia implica una reducción de los cuerpos de agua en los salares.

El salar de Surire se localiza en el norte de Chile a $18^{\circ} 53^{\prime} \mathrm{S}$, y $69^{\circ} 03^{\prime} \mathrm{O}$, a una altitud media de $4300 \mathrm{~m}$. Este cuerpo evaporítico forma parte de un conjunto de sistemas salinos que se desarrollaron durante el Pleistoceno y Holoceno en una amplia región de Sudamérica. El salar está totalmente rodeado de volcanes con una altitud promedio de $5500 \mathrm{~m}$. La influencia del vulcanismo en la formación y evolución de la cuenca está evidenciada por varias características, entre las que destacan la presencia del volcán Oquecollo en el centro del salar, y la existencia de surgencias termales en el sector suroriental.

Los salares se formaron por la progresiva acumulación de sales precipitadas en lagos de elevada salinidad, pero las condiciones climáticas reinantes actualmente en la región condicionan que éstos aparezcan como extensiones cubiertas por material salino, donde la presencia de agua se limita a puntos de aportes provenientes de vertientes y surgencias termales o zonas de acumulación de aguas meteóricas.

El clima del área de interés queda caracterizado con el denominado "clima de tundra por efecto de altura con precipitación estival", el cual se manifiesta en la zona cordillerana del extremo norte del país. La temperatura mínima absoluta oscila entre $0{ }^{\circ} \mathrm{C}$ y $13{ }^{\circ} \mathrm{C}$ (Junio a Septiembre). La temperatura máxima mensual absoluta oscila entre $18{ }^{\circ} \mathrm{C}$ y $20{ }^{\circ} \mathrm{C}$ (Diciembre a Enero). En general, existe una marcada diferencia de temperatura entre el día y la noche, con una amplitud del orden de $15{ }^{\circ} \mathrm{C}$. Las precipitaciones registradas son del orden de $200 \mathrm{~mm} / \mathrm{año} \mathrm{y} \mathrm{se}$ concentran en el periodo estival en que cae el 70 $\%$ de la precipitación anual.

El análisis sedimentológico y estructural de las unidades sedimentarias más antiguas demuestra la existencia de un paleolago de mayor extensión que la actual cuenca evaporítica con un desarrollo afectado por una intensa actividad volcánica. El relleno inicial de la cuenca correspondió a material clástico en el seno de un lago que, en el transcurso del tiempo geológico y a consecuencia de las variaciones climáticas, evolucionó a lago salino (Garcés y López, 2005). Los $60 \mathrm{~m}$ del relleno superior del salar lo componen materiales evaporíticos, sedimentos orgánicos y rocas sedimentarias de grano fino, diferenciándose dos unidades, una inferior en que predominan sedimentos ricos en materia orgánica y con una fina diseminación de sales (sulfatos, cloruros y boratos), y otra superior constituida principalmente por sulfatos, cloruros, boratos y carbonatos subordinados, en que se incluye la cubierta actual del salar.

La alimentación hídrica actual del salar proviene mayoritariamente de los aportes que canalizan las precipitaciones que caen sobre la cuenca de drenaje $\mathrm{y}$ de los aportes subterráneos que se manifiestan como surgencias termales. Una parte de las precipitaciones caen directamente sobre la superficie del cuerpo salino, y a causa del ligero basculamiento del mismo tienden a circular hacia el sector occidental, donde se acumulan dando lugar a lagunas salinas estacionales. El resto de las precipitaciones se infiltran en los niveles de materiales clásticos que rellenan las vertientes, y acceden al salar como afloramientos de agua con muy baja concentración en solutos. Es el caso por ejemplo del río Surire, que drena un área de unos $80 \mathrm{~km}^{2}$ en el sector sur de la cuenca, pero cuyo acceso al 
salar tiene lugar de manera difusa y no como un curso superficial de agua bien canalizado.

El otro aporte principal de agua tiene lugar a través de surgencias termales, que se localizan en el sector sureste del salar. De ellas la más importante es la de Polloquere, que aflora entre los materiales salinos del salar con un caudal aproximado de $50 \mathrm{~L} / \mathrm{s}$ y una temperatura de surgencia en torno a $80{ }^{\circ} \mathrm{C}$ (Garcés y López, 2005). El agua aportada por las surgencias termales da lugar a un curso de agua que fluye sobre la costra del salar hacia el oeste, incrementando progresivamente su contenido en solutos por disolución de los materiales salinos sobre los que discurre.

Un aspecto relevante de la hidrología del salar de Surire es la existencia de una aureola pantanosa rica en vegetación, que se dispone en el área marginal del cuerpo salino que constituye el salar. Se trata de un ecosistema especial conocido como bofedal y que consiste en un humedal de altura donde se desarrolla variada y abundante vegetación. Este ecosistema convive en medio de una gran extensión de sales, principalmente boratos que inciden en el desarrollo de su vegetación, y otras como arsénico que le confieren cierto carácter tóxico. La importancia de estos bofedales es el agua que contienen y que es esencial para la variada biodiversidad existente en el salar.

\section{METODOLOGÍA}

A partir del estudio realizado por Garcés y López (2005), en que se definieron criterios de elección de puntos muestreados, para soluciones superficiales en las áreas de aportes a vertientes y surgencias termales, como en las de acumulación en los sectores marginales (bofedales) y más internos (láminas de salmuera); y en el que se caracterizaron químicamente los principales cuerpos de agua del salar, y las relaciones químicas existentes entre ellos; unido al trabajo de Garcés et al. (2009) en el que a partir de datos metereológicos, aguas lluvias y temperaturas (adquiridos a la Dirección General de Aguas) se predice el cambio del recurso hídrico en la cuenca y sus aportes, se tiene la información base que sustenta el presente trabajo.

Las muestras de aguas que se tomaron en distintas estaciones del año, cubren un rango amplio de las condiciones naturales bajo las cuales evoluciona el sistema salino. De forma simultánea a la recogida de muestras se determinaron in situ la temperatura y el $\mathrm{pH}$ de las soluciones, procediéndose a almacenar las muestras al abrigo de la luz durante su traslado al laboratorio de análisis químico, donde se realizaron las determinaciones del contenido en $\mathrm{Ca}, \mathrm{Mg}, \mathrm{Na}, \mathrm{K}, \mathrm{Li}$ (todos ellos mediante espectrometría de absorción atómica), $\mathrm{SO} 4$ (precipitación con $\mathrm{BaCl}_{2}$ ), $\mathrm{Cl}$ (método de $\mathrm{Mohr}$ ), $\mathrm{HCO}_{3}$ y $\mathrm{CO}_{3}$ (potenciometría) y, en algunas muestras, se analizó también su contenido en B (técnica ácido-base).

Durante las campañas al salar, se tomaron fotografías a las comunidades vegetales y se reconocieron algunas aves y mamíferos, las que posteriormente fueron contrastadas con fuentes directas. A partir de estos resultados se realiza un análisis desde el punto de vista de la biodiversidad que presenta el salar y sus consecuencias que podrían existir producto de las sequías y del aumento de la temperatura.

\section{RESULTADOS Y DISCUSIÓN}

Se presenta un resumen de la información recopilada de una campaña en terreno que permitió caracterizar el medio biótico, abarcando vegetación, flora y fauna.

\section{Marco biogeográfico del área de estudio}

Debido a que la distribución de la vegetación está determinada por la altitud $\mathrm{y}$, por consiguiente, depende del clima en la medida en que aumenta la disponibilidad de agua $y$ disminuye la temperatura media anual, pueden distinguirse diversos tipos biológicos como: suculentas y leñosas bajas (cactus y arbustos) leñosas bajas (arbustos), herbáceas (coirones y arbustos y pastos de tundra). La comunidad vegetal más típica la constituyen los "Pajonales", con paja brava (Festuca orthophylla), huailla (Deyeuxia breviaristata), extensas superficies 
cubiertas por gramíneas en mechón, que se encuentra principalmente en los llanos arenosos, de mayor altitud. Se distingue la Tola como la especie más frecuente, los "tolares", con tola (Parastrephia quadrangularis) -paja brava (Festuca orthophylla), y los "llaretales", característicos por la dominancia de la forma de vida pulvinada de la Azorella compacta.

En los cursos de agua se encuentra una agrupación vegetal que recibe el nombre genérico de "Bofedal", dominada por paquial (Oxychloe andina), y que en publicaciones se cita como complejo florístico y vegetacional.

Según Marticorena (1990), la flora potencial del área alcanzaría unas 57 especies. La Tabla 1, resume las especies encontradas en este. Es necesario destacar que de todas las especies encontradas, la Azorella compacta ("Llareta"); y Ephedra breana (pingo pingo), son consideradas como vulnerables a nivel regional y nacional.

Tabla 1 Vegetación del salar de Surire.

\begin{tabular}{ll}
\hline \multicolumn{1}{c}{ Especie } & \multicolumn{1}{c}{ Nombre Cientifico } \\
\hline TOLA & Parastrephia \\
QUEÑOA DE & Polylepis tarapacana \\
ALTURA & Fabiana densa \\
TOLA & Polylepis rugulosa \\
QUEÑOA & Senecio nutans \\
CHACHACOMA & Oxychloe andina \\
BOFEDAL & Azorella compacta \\
LLARETA & Opuntia ignescens \\
PUSCAYA & Festuca ortophylla \\
\hline
\end{tabular}

De estudios previos (Muñoz y Bonacic 2006), quienes constataron que se produce un cambio proporcional de cobertura vegetal entre estaciones húmedas (año 2002) y secas (2002). Entre la diversidad vegetal, las terófitas o hierbas anuales, son la comunidad de plantas más afectada por la disminución de las precipitaciones, éstas no sólo son sensibles a la disponibilidad hídrica, sino que, además, se ve amenazada por el aumento de la masa de ganado introducido en los últimos años en el área.

\section{Fauna}

El entorno del salar constituye el hábitat de un número considerable de especies de mamíferos silvestres, dentro de los cuales destacan por su abundancia la Vicuña, la Vizcacha y el Zorro Culpeo. Según Glade (1993), las especies Puma, el Gato Colocolo y el Quirquincho se encuentran clasificados, tanto a nivel nacional como regional, como especies en peligro de extinción. Por otro lado, la Vicuña se encuentra clasificada como una especie vulnerable a nivel nacional, mientras que a nivel regional se encuentra fuera de peligro. Respecto a ejemplares de Quirquinchos en el área, las prospecciones realizadas permiten identificar pequeñas colonias ubicadas en la zona Altos de Paquiza en el sur de la cuenca Surire, esta especie se encuentra escasamente representada en parques $\mathrm{y}$ reservas nacionales.

Las lagunas existentes en el salar, constituyen el hábitat para numerosas aves adaptadas a condiciones de altura, destacándose la existencia permanente de tres especies de flamencos de los seis existentes en el mundo, los que se alimentan del plancton, diatomeas, invertebrados bentónicos como copépodos, anfípodos, artemias salinas y dunaliellas, existentes en las lagunas salinas. Por su parte, las aguas termales del sector sur del salar, originan una laguna de agua dulce en que habitan varias especies de aves. Las especies presentes en el área de estudio que se asocian exclusivamente a bofedales y vertientes de agua dulce son la Guallata, Pato jergón del norte, Pato juarjual, Tagua gigante.

De todas las especies de aves identificadas que habitan el salar, el Suri se encuentra tanto a nivel regional y nacional como especie en peligro de extinción y la Perdicita Cordillerana como una especie rara, es decir, muy difícil de encontrarla con frecuencia, mientras que el Kiula, el Cóndor, la Tagua gigante y las tres especies de flamencos se encuentran clasificadas como especies vulnerables desde el punto de vista de su estado de conservación, tanto a nivel nacional como regional. 
La región en que se encuentra el salar, posee un $45 \%$ de las especies de vertebrados descritas para la bioregión de Praderas Altomontañas, que comprende áreas de Perú, Bolivia, Argentina y Chile y un $99 \%$ de las especies de aves, mamíferos, reptiles y anfibios descritos para la bioregión en Chile, lo que implica que esta región representa un área de gran concentración de biodiversidad, calificando como un "Areacaliente" o "Hot-spot" de biodiversidad a nivel nacional y regional. La Tabla 2 resume una actualización de la avefauna existente en el salar, la cual se ve amenazada por la falta de agua.

En trabajos previos (Garcés et al., 2009), la relación existente entre las surgencias termales y las salmueras por un lado, y entre las aguas de vertientes y de bofedales por otro, indican la falta de relación directa entre ambos grupos de soluciones. Conclusión relevante para un ecosistema muy rico en vida animal y vegetal, que se alimenta exclusivamente por aguas meteóricas muy diluidas, y cuya existencia está condicionada por la falta de relación directa con las aguas que circulan por la superficie del salar, caracterizada por presentar una concentración iónica mucho mayor (por tanto perjudicial para el desarrollo de vida, como se observa en el sector interno del salar) y unas relaciones iónicas muy diferentes (datos de composiciones químicas de las aguas en Garcés y López, 2005).
El arsénico y boro se encuentran con frecuencia en la zona asociado al volcanismo cuaternario de la zona altiplánica, las aguas presentan concentraciones altas de As de $147 \mathrm{mg} / \mathrm{L}$ y en boro $1600 \mathrm{mg} / \mathrm{L}$. La incorporación del As y B al ciclo hidrológico en la zona, tiene su origen en la actividad volcánica y en los depósitos evaporíticos.

Por otra parte, desde el punto de vista mineralógico, el único mineral económico reconocido mediante difracción de rayos $\mathrm{X}$ corresponde a ulexita y su equivalente deshidratado, probertita. Este se encuentra asociado a materiales volcánicos detríticos (feldespato, vidrio, minerales máficos), yeso, y silicatos de alteración. Las leyes en la ulexita varían entre 8 y $36 \% \mathrm{~B}_{2} \mathrm{O}_{3}$. Los contenidos en litio van entre 20 y $400 \mathrm{ppm}$, valores relativamente altos, además, se han encontrado anomalías en estroncio.

Un resumen de las precipitaciones, Figura 1, muestran sus máximos en la década de los 80 , sin dejar de lado que se refleja una baja de precipitaciones en los siguientes años sobre todo en el siglo XXI. A partir del año 1986 se observa descenso de las lluvias, además que las precipitaciones en el altiplano se concentran en verano, esencialmente, en los meses de Enero y Febrero.

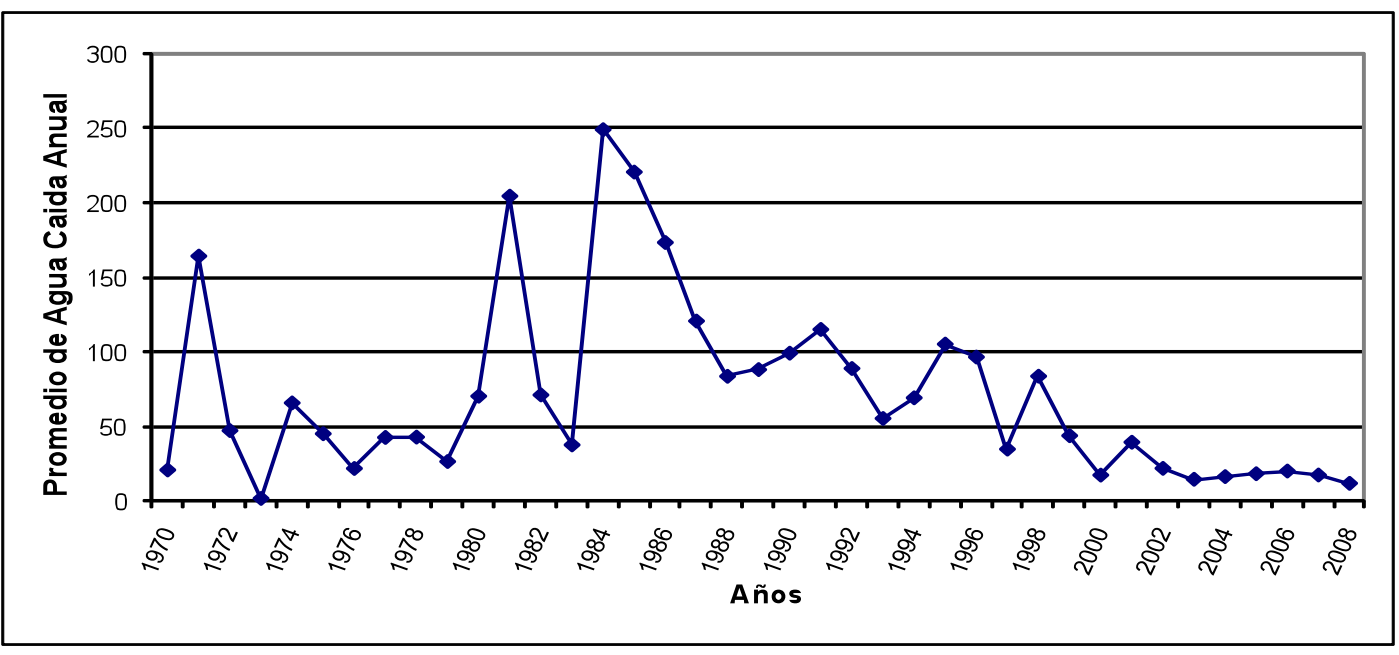

Figura 1 Promedios anuales de agua caída en mm, entre los años 1970 al 2008. 
Tabla 2 Avifauna existente en la cuenca del salar de Surire. Tomado y ampliado de Santoro y Nuñez (1985).

\begin{tabular}{|c|c|c|}
\hline Especie & Nombre Científico & Abundancia \\
\hline PERDIZ DE PUNA O KIULA & Tinamotis pentlandii & $\mathrm{MC}$ \\
\hline FLAMENCO ANDINO & Phoenicoparrus andinus & $\mathrm{MC}$ \\
\hline FLAMENCO CHILENO & Phoenicopterus chilensis & $\mathrm{MC}$ \\
\hline FLAMENCO DE JAMES & Phoenicoparrus jamesi & $\mathrm{MC}$ \\
\hline PATO JUARJUAL & Lophonetta specularioides alticola & $\mathrm{C}$ \\
\hline CUERVO DEL PANTANO & Plegadis Ridgwayi & \\
\hline ÑANDÚ O SURI & Pterocnemia pennata tarapacensis & $\mathrm{MC}$ \\
\hline GUALLATA O PIUQUEN & Chloephaga melanoptera & $\mathrm{MC}$ \\
\hline CONDOR & Vultur gryphus & $\mathrm{E}$ \\
\hline CAITI & Recurvirostra andina & $\mathrm{MC}$ \\
\hline AGUILUCHO & Buteo polyosoma & $\mathrm{C}$ \\
\hline AGUILUCHO DE LA PUNA & Buteo poecilochrous & $\mathrm{R}$ \\
\hline TIUQUE CORDILLERANO & Phalcoboenus megalopterus & $\mathrm{C}$ \\
\hline HALCÓN PERDIGUERO & Falco femoralis pichinchae & $\mathrm{C}$ \\
\hline HALCÓN PEREGRINO & Falco peregrinus & $\mathrm{C}$ \\
\hline TUCUQUERE & Bubo magellanicus & $\mathrm{E}$ \\
\hline CHORLO DE CAMPO & Oreopholus ruficollis & $\mathrm{C}$ \\
\hline CHORLO DE LA PUNA & Charadrius alticola & $\mathrm{C}$ \\
\hline GAVIOTA ANDINA & Larus serranus & $\mathrm{C}$ \\
\hline PLAYERO DE BAIRD & Calidris bairdii & $\mathrm{C}$ \\
\hline POLLITO DE MAR ROJIZO & Phalaropus fulicarius & $\mathrm{C}$ \\
\hline PERDICITA CORDILLERANA & Attagis gayi gayi & $\mathrm{C}$ \\
\hline PERDICITA COJÓN DEL NORTE & Thinocorus orbignyanus ingae & $\mathrm{MC}$ \\
\hline TORTOLITO DE LA PUNA & Metriopelia aymara & $\mathrm{MC}$ \\
\hline TORTOLA CORDILLERANA & Metriopelia melanoptera & $\mathrm{C}$ \\
\hline TORTOLITA BOLIVIANA & Metriopelia ceciliae gymnops & $\mathrm{C}$ \\
\hline MINERO & Geositta cunicularia titicacea & $\mathrm{MC}$ \\
\hline MINERO DE LA PUNA & Geositta punensis & $\mathrm{MC}$ \\
\hline BANDURRILLA DE LA PUNA & Upecerthia jelskii & $\mathrm{C}$ \\
\hline PATO JERGÓN CHICO DEL NORTE & Anas flavirostris oxypterum & $\mathrm{MC}$ \\
\hline JILGUERO NEGRO & Carduelis atratus & $\mathrm{C}$ \\
\hline COLEGIAL & Lessonia rufa oreas & $\mathrm{E}$ \\
\hline GAUCHA O MERO DE TARAPACA & Agriornis montana maritima & $\mathrm{C}$ \\
\hline MERO DE COLA BLANCA & Agriornis albicauda & $\mathrm{R}$ \\
\hline TAGUA GIGANTE & Fulica gigante & $\mathrm{E}$ \\
\hline VICUÑA & Vicugna vicugna mensalis & $\mathrm{MC}$ \\
\hline ALPACA & Lama pacos & $\mathrm{C}$ \\
\hline VIZCACHA & Lagidium viscacia cuvieri & $\mathrm{MC}$ \\
\hline GATO COLOCOLO & Lynchailurus colocolo garleppi & $\mathrm{E}$ \\
\hline CHINGUE REAL & Conepatus chinga rex & $\mathrm{E}$ \\
\hline ZORRO CULPEO & Pseudalopex culpaeus andinus & $\mathrm{C}$ \\
\hline LLAMA & Lama glama & $\mathrm{C}$ \\
\hline QUIRQUINCHO & Euphractus nationi & $\mathrm{R}$ \\
\hline TUCO TUCO DE LA PUNA & Ctenomys opimus & $\mathrm{E}$ \\
\hline CUY DE LA PUNA & Galea musteloides & $\mathrm{C}$ \\
\hline PUMA & Puma concolor incarum & E \\
\hline
\end{tabular}

Clave Abundancia: MC: Muy común; E: Escaso; C: Común; R: Raro 


\section{CONCLUSIONES}

En general, en el altiplano las condiciones climáticas imperantes y las características del suelo determinan una precaria disponibilidad hídrica en el área estudiada. El bofedal de Surire que se ubica por encima de los 4300 m.s.n.m., de origen natural y de tipo hidromórfico o údico, se ha presentado empapado en agua casi de manera permanente, existiendo abundante y variada vegetación con comunidades de animales muy bien representadas. Pero a partir del presente estudio se prevé que la disponibilidad de los recursos hídricos en los siguientes años será cada vez menor en el marco del cambio climático, las que se asocian directamente con el patrón de precipitaciones en el altiplano considerando que el principal aporte hídrico que sustentan los humedales corresponde a las lluvias. Como consecuencia del menor aporte de agua y el aumento de la temperatura, se observa una clara amenaza a la existencia de bofedales que mantienen la fauna y flora de esta región andina.

\section{AGRADECIMIENTOS}

La autora agradece a la Universidad de Antofagasta por el soporte financiero a esta investigación. Agradecer a los árbitros que de forma anónima han corregido y mejorado este artículo.

\section{REFERENCIAS}

Garcés, I., Alvarez, G. \& Reyes, J. (2009). Cambio climático y su incidencia en el salar de Surire. Modelos para el análisis de datos para aguas lluvia y temperaturas usando STATGRAPHICS. Actas del 9no Congreso Interamericano de Computación Aplicada a la industria de Procesos (CAIP 2009). Montevideo, Uruguay.

Garcés, I. \& López, P. (2005). Hydrochemistry of Springs, Wetlands, and Surface Brine Ponds of the Surire Salt Deposit (Chile). In Proceedings of the 2nd Southern Deserts Conference. Arica, Chile.
Glade, A. (1993). Libro Rojo de los Vertebrados Terrestres de Chile. Corporación Nacional Forestal. Santiago, Chile.

Honty, G. (2007). América Latina ante el cambio climático. El Observatorio de la Globalización (OG). D3E. París, Francia. Disponible en [consulta: 13 de Junio de 2011]: http://www.ecosistemas.cl/1776/articles77401_Documento_pdf.pdf

Marticorena, C. (1990). Contribución a la estadística de la flora vascular de Chile. Gayana Botánica, Vol. 47, No. 3-4, pp. 85-113.

Muñoz, A. \& Bonacic, C. (2006). Variación estacional de la flora y vegetación en la precordillera andina de la comuna de Putre (I Región de Tarapacá, Chile) durante el período 2002-2003. Gayana Botánica, Vol. 63, No. 1, pp. 75-92. doi:10.4067/S0717-66432006000100003

Santoro, A. \& Nuñez, E. (1985). Guía de Manejo de Monumento Natural del Salar de Surire. Conaf I Región. Arica, Chile.

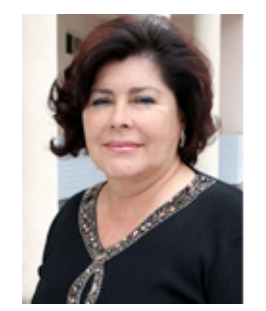

Ingrid Garcés, Ingeniera Civil Química, Doctora en Ciencias de la Universidad de Zaragoza España, se desempeña como académica en el Departamento de Ingeniería Química y en el Centro de Estudios y Educación Ambiental, en las cátedras de minerales industriales y desarrollo sustentable, depósitos salinos andinos, termodinámica de soluciones salinas, industrias químicas. $\mathrm{Su}$ área de investigación es medio ambiente con énfasis en depósitos salinos andinos y minerales industriales. 